\title{
Image Plane
}

National Cancer Institute

\section{Source}

National Cancer Institute. Image Plane. NCI Thesaurus. Code C102516.

The position and orientation of the image slice relative to the patient-based coordinate system. 